\title{
Observations of Surface Changes in Platinum Crucibles
}

\author{
Charles Proffer Saylor, Edward Wichers, and James I. Hoffman* \\ National Measurement Laboratory, National Bureau of Standards, Washington, DC 20234 \\ June 6, 1979
}

\begin{abstract}
Iron which has been in solid solution in a platinum crucible will cause iron determinations in analyses to become high. Likewise the iron, oxidizing to ferric oxide during ignition, segregates along the boundaries between platinum crystals. It causes embrittlement and eventual crumbling of the crucible.

During these studies it was observed that after heating in an electric muffle furnace the surface of a crucible was covered by tiny crystallographic faces. When, however, heating to the same temperature was carried out in a gas flame the facets almost smoothed out of existence.
\end{abstract}

Key words: Contrast augmentation of reflecting surfaces; double-diaphragm; faces (crystal) on platinum crucibles; hematite forming on platinum; iron as alloy in platinum; platinum ware; surface of platinum influenced by method heating.

\section{Historical Introduction}

For about fifty years spanning the last quarter of the nineteenth and the first quarter of the twentieth centuries, geochemistry stood very high on the scale of importance in the field of chemical research. Extraordinary demands were made on accuracy in the chemical analysis of the silicate and carbonate rocks that constitute most of the earth's crust.

The late William Francis Hillebrand $[1]^{1}$ was internationally recognized as one of the foremost practioners in the field of rock analysis [2]. When Hillebrand, in 1909, left the U. S. Geological Survey to become Chief Chemist of the National Bureau of Standards, he retained his interest in rock analysis and his awareness of the hindrances to perfection in the art stemming from impurities in reagent chemicals and the shortcomings of platinum utensils, which were essential tools of the rock analyst. He launched efforts toward the improvement of reagent chemicals which led to the creation of an American Chemical Society Committee on Reagent Chemicals. The committee has had a continuous productive life to the present, with excellent cooperation from the reagent-manufacturing industry.

In his efforts to bring about improvements in the quality of platinum laboratory ware, Hillebrand sought similar cooperation from the platinum industry but met with so little success that he found it necessary to initiate in 1917 an independent program dealing with the properties of each of the platinum-group metals in highly purified form and with analytical procedures for alloys or mixtures of these elements.

\footnotetext{
* Deceased.

${ }^{1}$ Figures in brackets indicate literature references at the end of this paper.
}

Some of the faults that Hillebrand had observed in commercially available platinum utensils were lack of constancy of weight following heating at the requisite temperatures, brittleness, intercrystalline cracking and, on occasion, surface alkalinity after ignition.

It soon became evident that the platinum commonly used for the commercial fabrication of utensils was in fact an alloy of platinum with varying proportions of other platinum-group metals, principally iridium and rhodium. It usually contained variable small amounts of iron and other impurities under special circumstances to be mentioned later. The usual total content of such extraneous alloying constituents was seldom above 1 percent.

In a study by Burgess et al. [3] conducted prior to the initiation of the research program, it was found that all platinum alloys except those containing gold are thermoelectrically positive to pure platinum. The observed thermal emf could serve as a means of classifying commercial platinum ware in terms of an apparent content of alloying elements, usually rated as iridium since this was the most abundant secondary element in native platinum. The hot junction was easily made by spot welding the reference platinum wire to the utensil or merely attaching it with a clip. This classification proved useful, in correlation with other observed characteristics, for judging the qualities of the ware for laboratory service. Such judgment became significant when it was noted that crucibles containing more than about 1 percent of iridium lost more weight on ignition than those made of a purer grade of platinum or an alloy containing rhodium. It was inexpedient to eliminate the alloying element completely because the highly purified metal is too soft to withstand the deformation of a utensil, 
especially a crucible, in its normal use. Thus for a time platinum utensils were procured under a requirement that they contain 0.75 percent of iridium as measured by the thermal emf test described above. At the time it was impractical to require rhodium as the alloying constituent. The problem of inconstancy of weight on ignition, which was not completely solved by this approach to improving the quality of platinum utensils, will be further referred to later in this paper.

Learning how to purify platinum to the highest possible degree was an essential part of the entire investigation. At first, the scheme of purification followed the traditional procedure of repeated precipitation of the slightly soluble salt ammonium chloroplatinate and ignition of the salt to platinum "sponge." The progress of purification was followed by melting small portions of the successive lots of sponge and rolling the resulting button to a thin ribbon whose thermal emf against a reference specimen was then measured. In the early stages of the operation, successive samples always showed a declining thermal emf. But after several such steps the observed thermal emf became variable, sometimes indicating an apparent decrease in purity. The cause of this behavior, when discovered, led incidentally to the explanation of the surface alkalinity of platinum utensils sometimes observed after ignition.

The commercial practice for melting platinum and casting it into ingots suitable for rolling to sheet or drawing to wire involved the use of an oxygen-hydrogen torch and a crude crucible made by shaping a cavity in a block of commercial quicklime (calcium oxide). This was the practice followed in producing the small sample buttons of metal that were then rolled to ribbons suitable for thermoelectric comparison with the reference specimen. It was soon noted that in some cases the molten metal moved freely over the lime surface under the force of the flame and congealed to a button that showed little or no adhesion to the lime but that other samples seemed to "wet" the surface and to adhere to it after cooling.

When it was found that the "wetting" phenomenon correlated with higher thermal emf the inference was drawn that the adhering specimens contained an impurity derived from the calcium oxide base on which it was melted and that the impurity presumably was calcium. This inference was supported by the flash of red that appeared when the end of the rolled-out ribbon was melted in the flame of the oxyhydrogen torch. It was also observed that changing the composition of the oxygen-hydrogen mixture fed to the flame from oxygenrich to hydrogen-rich resulted in a change from non-adhering to adhering buttons. Thus it appeared, unexpectedly, that calcium oxide was being reduced locally in contact with molten platinum with the resulting formation of a platinumcalcium alloy. This conclusion was confirmed by producing a series of such alloys of varying calcium content as determined by chemical analysis, with corresponding varia- tions in thermal emf toward a reference specimen. When the calcium-contaminated specimens were strongly heated in air their thermal emf gradually fell toward zero, indicating that the calcium was either diffusing out of the alloy or was being oxidized in place by oxygen diffusing into the heated metal.

From these simple observations it was reasonable to conclude that a platinum utensil which on heating acquired an alkaline surface deposit had been fabricated from platinum melted in an oxygen-deficient flame. By analogy the embrittlement of platinum crucibles in which magnesium ammonium phosphate was converted to magnesium pyrophosphate (the form employed in the determination of magnesium in rock analysis) by the flame of a Bunsen-type burner was the result of hydrogen-reduction of the ignited residue in contact with its platinum container.

The obvious remedy for this problem was to substitute an electrically heated furnace. Electrically heated furnaces, however, introduced a new problem for the analyst. If the heating element was made of the commonly used nickelchromium alloys, a platinum crucible was separated from the hot resistance wire only by a porous refractory shell. Sometimes the crucible would gain weight, presumably by transfer of metal vapor through the refractory wall.

The historical introduction given in the preceding paragraphs, covering the period before about 1925, has never been published in any systematic fashion. It is presented here from recollections of the present authors (a sort of institutional memory) to be of assistance to the reader in understanding the observations and conclusions in the remainder of the present paper.

\section{High Iron Blanks}

Late in 1935 when one of us (J.I.H.) was concerned with some highly precise analyses for aluminum, he was troubled by unexpected persistently high blank determinations for iron. The precipitate caught upon an "ashless" paper and placed in a platinum crucible was ignited in an electric muffle furnace of which the heating elements were silicon carbide rods. The ash was extracted with acid and the amount of iron was determined. Each ignition and extraction yielded a new determination of iron, but if the strong ignition stage was omitted no iron was found. Bearing in mind the experience of the other workers in other years as described above it was decided to look for the source in the metal of the platinum crucible itself.

After such an ignition - four hours at $1200{ }^{\circ} \mathrm{C}$ in the muffle furnace - a commercially procured crucible was examined with a reflecting microscope. The entire surface was 


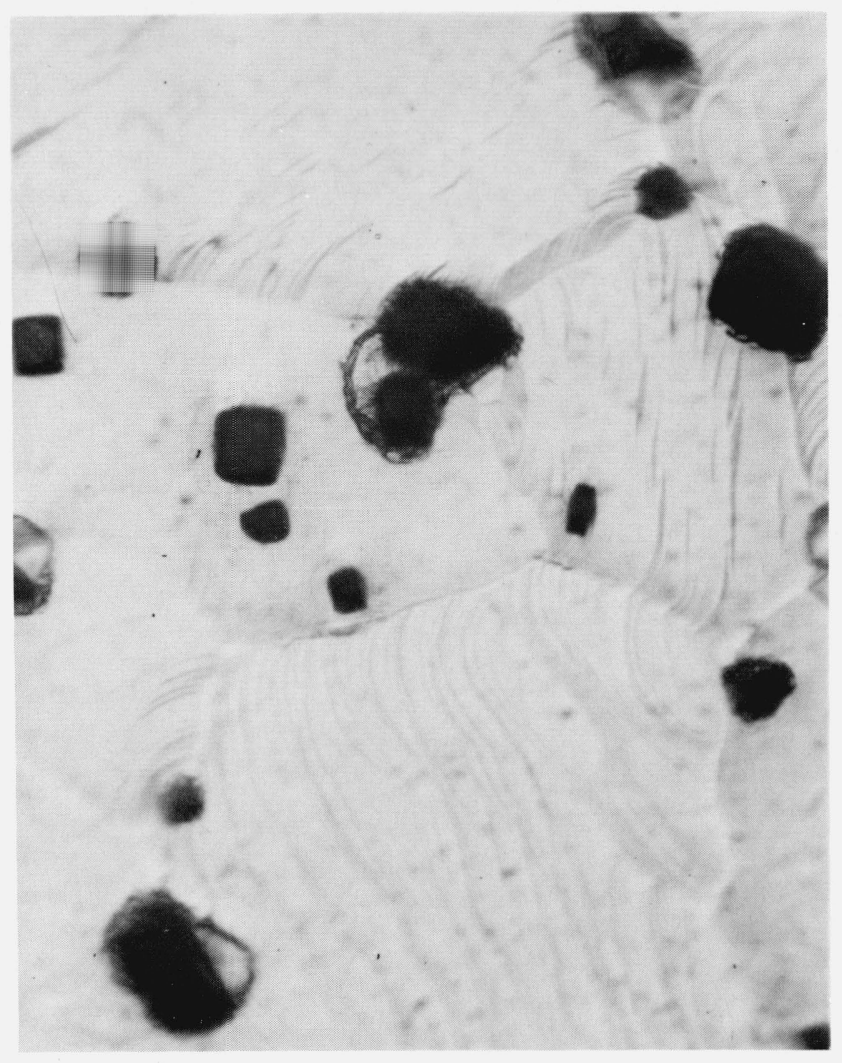

Figure 1. Dark spots on platinum crucible. $(\times 810)$

The crucible has been ignited at $1200{ }^{\circ} \mathrm{C}$ for four hours. The dark spots are oriented in accord with the surfaces on which they have formed, changing from metal crystal to crystal. Notice stripes in metal surface and boundaries between crystals. In general the spots are contained within cavities.

dotted with small, nearly black - possibly dark red - particles. Figure 1 shows a portion of the outside of the crucible. The picture was made with a $114 \mathrm{X}$ semi-apochromatic oilimmersion objective of $1.32 \mathrm{NA}$ and an $8 \mathrm{X}$ eyepiece. The direct magnification of about $912 \mathrm{X}$ was here photographically changed to $810 \mathrm{X}$. The spots tended to be aligned nearly parallel to each other and to faint stripes in the surfaces of the platinum. Near the center left of the photomicrograph is a triangularly shaped spot with truncated corners. Many of the spots seemed to be accompanied by irregularities in the adjacent surface of the platinum. In some cases it appeared as though a particle had shrunken and no longer completely filled the cavity within which it had formed.

Another commercial crucible subjected to the same treatment became covered with its own crop of dark spots. In this case most of the spots were three or six sided plates (fig. 2). This is fully consistent with figure 1 . If the spots have formed epitaxially on platinum faces, their orientation will have been determined by that of the underlying metal.

The crucibles were subjected to a normal extraction with hydrochloric acid, washing, and drying. Now, by microscop-

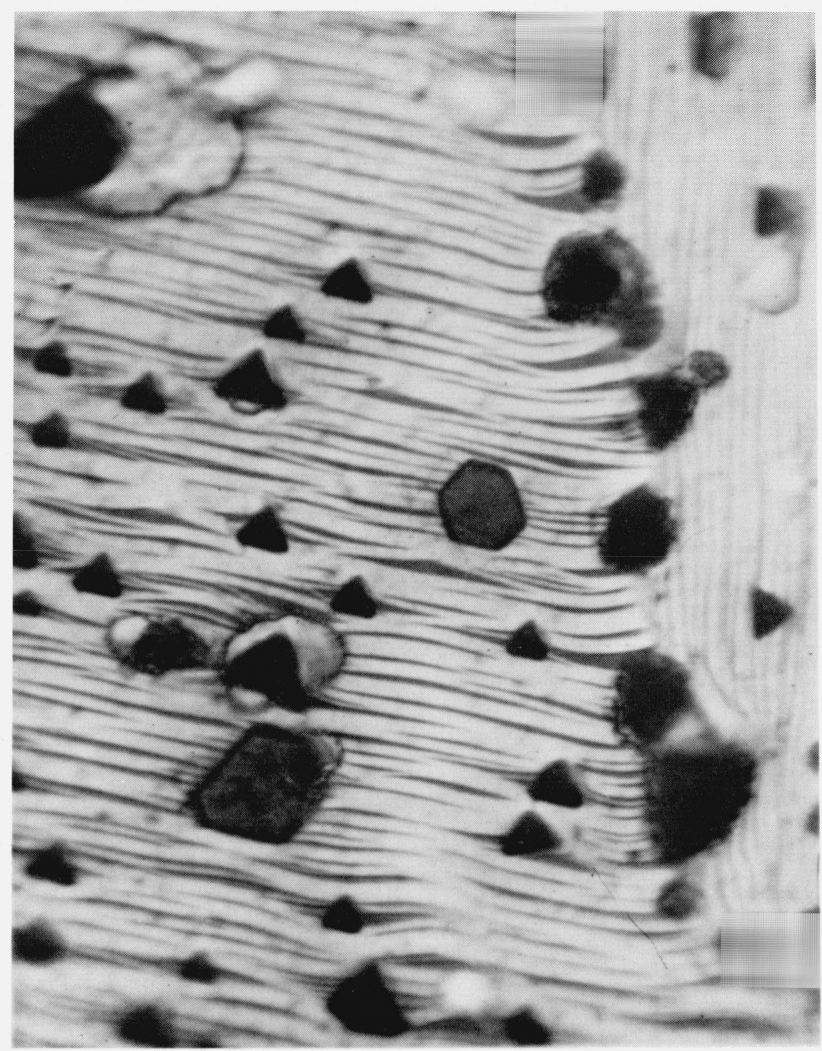

Figure 2. Another crucible after heating at $1200{ }^{\circ} \mathrm{C} .(\times 810)$

There are two metal crystals in the field, recognizable by the sets of stripes. In this case the spots are 3 or 6 sided having at least one side parallel to the stripes in the metal crystal.

ical examination, it was found that there were no dark spots. Many tiny cavities appeared where the dark spots had been; iron was contained in the extract.

The rather obvious conclusion was that the dark crystals were the source of the high iron blanks of the analytical work. The shape as shown in figure 2 made it practically certain that they were crystals. The concentration of the ironbearing crystals near the boundaries between the platinum crystals presumably was due to mobility of iron atoms within the platinum but restricted by the grain boundaries. Their subsequent oxidation yielded a product which attached to facets of the platinum crystals in an arrangement oriented in accord with the underlying surface. It was inferred that this was ferric oxide in the hematite form, which is stable at 1200 ${ }^{\circ} \mathrm{C}$. Although this intuitive identification of the spots seemed most likely to be correct, proof was difficult. Various tests were performed, all consistent with the idea of hematite, but none were totally conclusive.

Looking through the microscope there was a general feeling that the spots were red, but the surface of the platinum metal was very bright and the crystals were dark. 
Self deception would have been easy. We had expected the spots to be red. To darken the background without obscuring any reflected light from the spots, a small hole was cut through a sheet neutral filter of dyed gelatine. This was mounted between glass plates with a cement that nearly matched the refractive index of the gelatine. The assembled slab could be moved about in the image plane of a Wright eyepiece [4]. One by one the dark spots were viewed as surrounded and, in some cases, partly obscured by the darkened background caused by the fairly dense neutral filter. They were clearly deep red. Their absorption spectrum was compared with that of hematite fragments as mounted upon a reflecting metal surface. The spectra were observed with a microscope spectroscope of commercial manufacture [5]. The two kinds of spectrum were indistinguishable - the same sharp cut-off in the middle red region with rapidly increasing brightness toward the margin of the visible spectrum. It is probable that this sense of increasing brightness was enhanced by the non-uniform spreading of the spectrum by the optical instrument, dispersion being less at the long wavelength end of the visible spectrum.

Crystallographic attempts at identification of the phase of the dark red spots were somewhat inconclusive. The general habit of the crystals was correct but measurements of their birefringent refractive indexes by the immersion method were only partially successful. Because everything in sight was capable of standing high temperatures, both Merwin's selenium-sulfur mixtures [6] and Barth's thallium bromide-iodide mixtures [7] could be used as comparison materials for refractive index determination by the immersion methods. The first of these materials served to establish that all refractive indexes of all the dark red crystals were well above 2.32. The Barth mixed crystals were less useful in this case presumably because of the rigidities of the mixed thallium halide crystals. Thus the immersion mount would crack away from both the red crystals and the platinum surface during cooling. Merwin's selenium-arsenic selenide melts were not tried. While it was possible to say that all of the dark red crystals were much higher than 2.3 , a definitive value was out of the question. A number of factors contributed to this: the particles were close to the limit of resolution, the optics of comparisons by immersion where the particle studied is contained in a socket in a brightly reflecting metal were not adequately understood. The conditions are inherently difficult and not a part of normal practice. Taking into consideration the crystal shapes, their high refractive index, deep red spectrum, and quick solution in hydrochloric acid to give an iron-bearing solution, little doubt remained that the crystals were indeed ferric oxide in the hematite modification.

On this basis it was clear that the source of the iron blanks in the analytical procedure was the iron present as an impurity in the platinum from which the crucible had been fabricated. The next step in the study was to repeat the observations on a crucible formed from specially purified platinum metal of the quality used for platinum resistance thermometers and platinum, platinum-rhodium thermocouples. After heating in the furnace at $1200{ }^{\circ} \mathrm{C}$ for four hours, none of the dark spots appeared. This supported the concept that the dark red spots were hematite, formed from iron which had been in solid solution in the platinum of the commercial crucible and had diffused toward intercrystalline metal crystal boundaries or the surface where it oxidized to ferric oxide.

In further support of these general conclusions, a clean platinum crucible known to contain iron and the pure platinum crucible were heated together in a muffle at 1200 ${ }^{\circ} \mathrm{C}$. The dark reddish crystals appeared in great abundance over the surface of the iron-bearing crucible but none on the pure one.

The circumstances described above had a parallel in a study of the embrittlement of platinum crucibles containing iron which were repeatly heated to $1000{ }^{\circ} \mathrm{C}$ or higher. In such cases, the iron oxide crystals were even more highly concentrated along the boundaries between platinum crystals than they are in figure 2 .

\section{Alterations in the Surface of Pure Platinum Crucibles During Multiple Firings}

Attention had been given to the stripes which appeared on the surfaces of platinum crucibles. These were observed in both figures 1 and 2 , but the nature of the stripes was imperfectly disclosed. A microscopical viewing system was devised that would make it possible to interpret changes in surface orientation of the reflecting metal surface. This was based upon the double-diaphragm method [8] for observing refractile discontinuities in transparent suspensions, but it was altered to meet the requirements for vertical illumination, figure 3 . An approximately half diaphragm in the illuminating system blocks much of the light. Another diaphragm at the eyepoint (exit pupil) of the ocular intercepts most of the light that gets by. The entire field becomes a uniform gray unless local tilts in the reflecting surface direct light either toward or away from the ocular diaphragm. ${ }^{2}$

\footnotetext{
${ }^{2}$ Ideally, the diaphragms shown at positions 1 and 5 in figure 3 could be replaced by a single, nearly half-aperture diaphragm at position 2 . This would require a suitable metallographic objective in which the upper principal plane was above the back lens elements. The unobscured part of the aperture would, in its reflected image at 3 , be largely intercepted by the same diaphragm unless the beam had been deflected by slants in the surface of the object. Under the best conditions, if the diaphragm and its image were in the same plane, representations of surface tilts would be as good as those shown in figures 4,6 , and 7 . We did not find a fully satisfactory objective. The arrangement had the advantage that it was equally applicable to visual and photographic
} studies. 


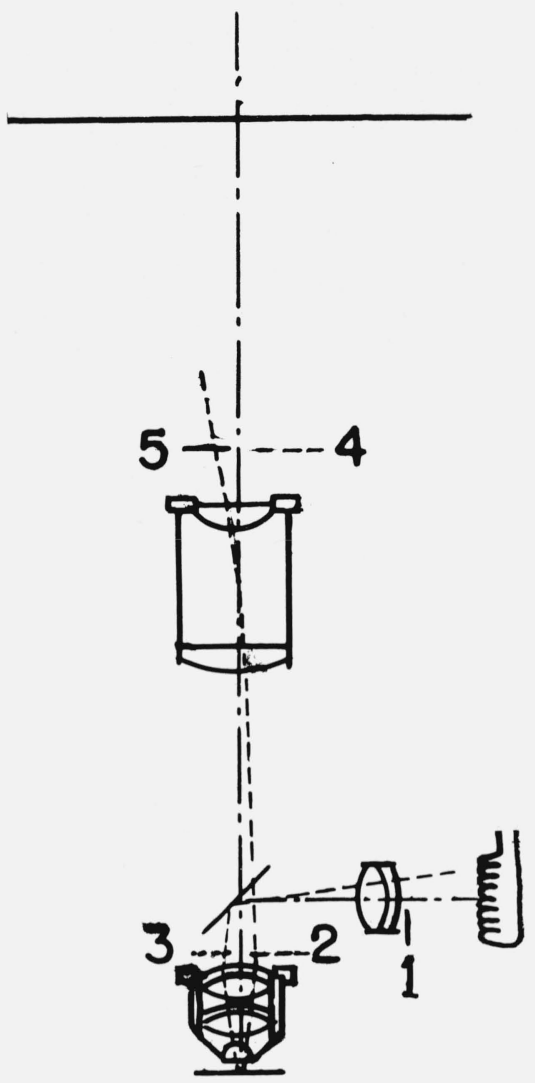

Figure 3. Special double-diaphragm conditions as adapted to vertical illumination and used for figures 4, 6, and 7 .

A little less than half the aperture is blocked by a diaphragm shown as a solid line at 1 . If the object is plane and reflecting, images of the diaphragm at 1 are represented by dotted lines at 2,3 , and 4 . A diaphragm at 5 , shown as a solid line is opposite the image 4 of the diaphragm at 1 , intercepts most, but not all, of the light reflected from the flat specimen. In each case actual diaphragms are shown as solid lines, their images as dotted lines. If there are tilts in the specimen the field is changed. A tilt to the left will divert light away from the diaphragm at 4 ; a tilt to the right will divert toward the blockage. In one case the region will appear brighter than the general fields; in the other case darker.

Figure 4 was made with this novel illuminating system. It is good for the reader who views this and figures 6 and 7 to sit so that his pages are seen by the light from a source to his right. This will give an illusion of slants in the surface but the illusion provides a correct impression of the true inclinations. The photomicrograph is strongly reminiscent of electron micrographs of shadow-cast surface plastic replicas. That totally different technique is based on different principles developed by Williams and Wyckoff [9] more than ten years after the photomicrograph (fig. 4) was made. In this figure outcroppings of four different platinum crystals are seen. A rough diagram of these areas is shown in figure 5 where the boundaries have been traced from figure 4 .

Although there are only four crystals, the surfaces of each of them are covered by a multitude of tiny crystal faces. We take it for granted that these are little octahedral surfaces.
By their tilt and frequency they unite to form the external surfaces of the crystals. The facelets are very small. Notice the serrations on the ridges of crystal 2. The distance between peaks is about $0.6 \mu \mathrm{m}$ and between the closest ridges about $0.5 \mu \mathrm{m}$. The complex of faces and corners is caused by mobility of the individual atoms of platinum which move around in the surface but do not evaporate because their velocity is too low for escape. They finally settle down into an approximation of the most stable configuration.

These surface effects demanded full attention. In chemical laboratory work, crucibles are heated either by muffle furnaces or in gas flames. To test different conditions of heating, the crucible was heated with a Meker burner. ${ }^{3}$ To achieve the same operating temperature, the primary air and

${ }^{3}$ The Meker is an adaptation of the Bunsen burner, designed to give a larger and more uniform region of high temperature. 


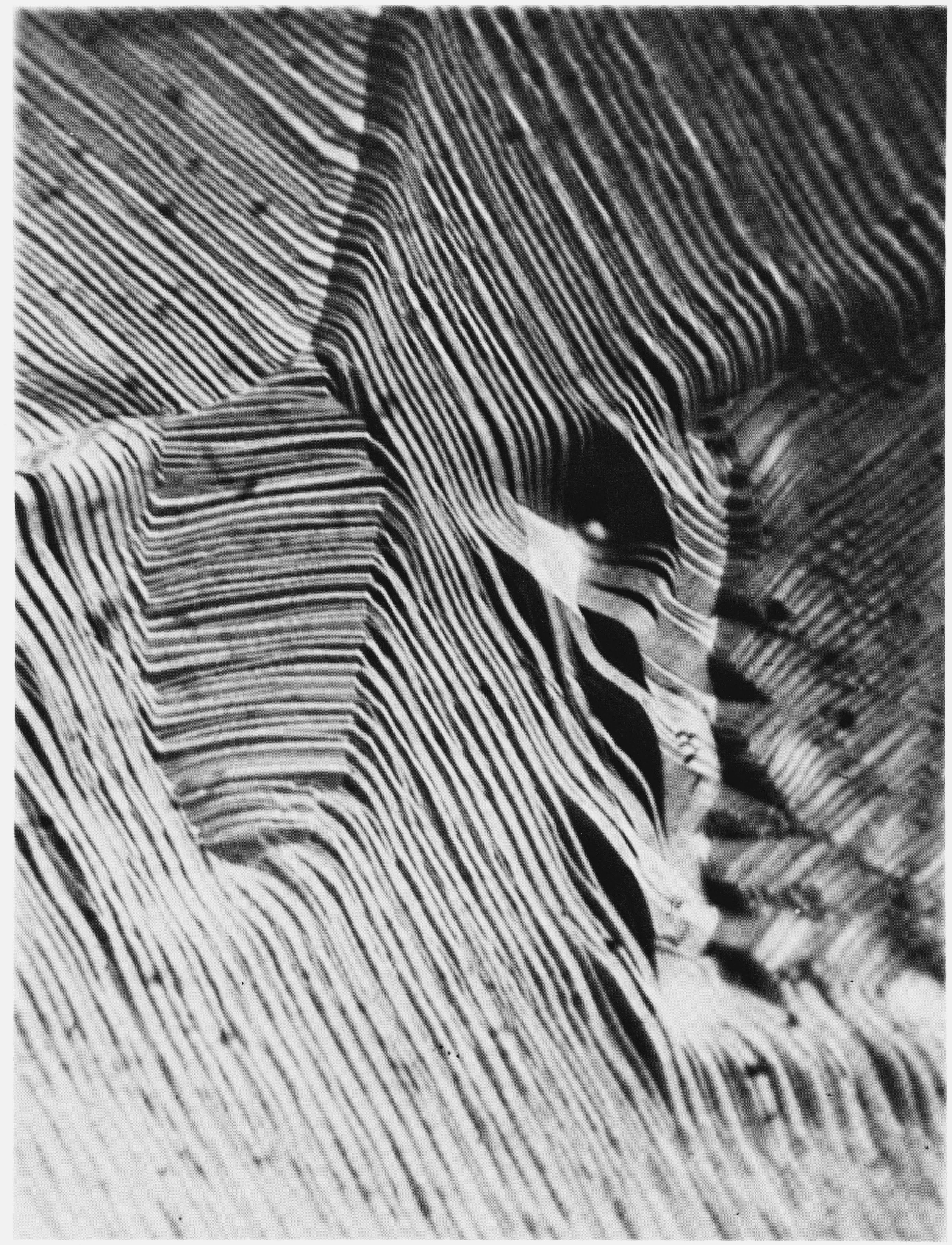

Figure 4. Pure platinum crucible heated in a silicon carbide muffle furnace four hours at $1200{ }^{\circ} \mathrm{C} .(\times 2000)$

Semiapochromatic 114X, N.A. 1.32 objective. Special diaphragming conditions as shown in figure 3 . Lines of contact between crystals are shown by furrows and by changes in direction of facelets. Some details - saw tooth facelets, facelets of low height or narrow width, etc. - lie close to the limit of optical resolution. They tend to be unnoticed in a contact print. 


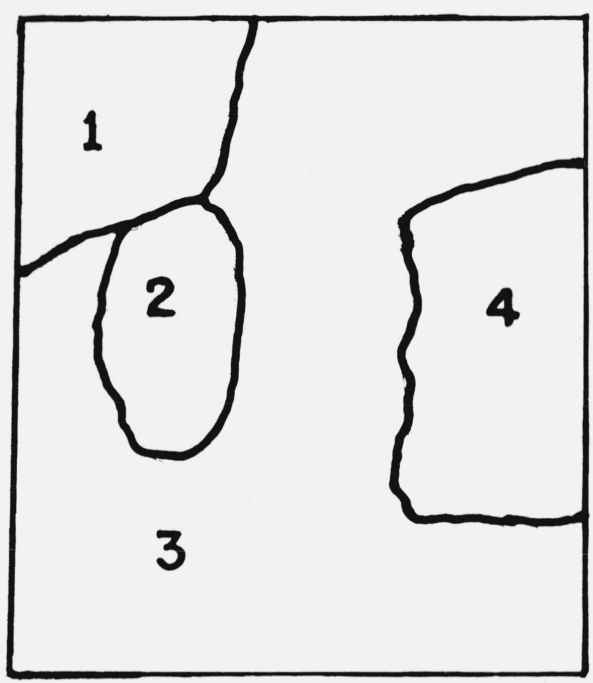

FigURE 5. Trace of crystal outlines of figure 4.

gas controls were manipulated sensitively and the temperature of the crucible evaluated with an optical pyrometer.

After four hours at a carefully controlled $1200{ }^{\circ} \mathrm{C}$, the same area on the crucible from figure 5 was as shown in figure 6. Part of the interface between crystal 2 and crystal 3 has an interesting puckered pattern. The original four crystals are still present in approximately their same positions. But another crystal, not previously seen, has grown into the domain of crystal 1. The complex pattern of octahedral faces has disappeared or almost so. One can see at some places on crystal 3 faint, closely spaced lines which are paraliel to the bold ridges shown on that crystal in figure 4 . This new one is close to the extreme left edge of the crystal. It would appear that there have been two or more stages in the migration of this intercrystalline boundary. It came so far, formed a furrow in the surface, and then moved on a very short distance.

The surface differences brought about when the crucible was heated in a gas flame instead of an electric furnace were perplexing. The gas flame indicated a much more labile condition of the metal surface during heating. The first hypothesis considered was that the actual temperature of the surface in the gas flame as monitored by an optical pyrometer had been different, probably higher, than with the thermocouple used to control temperature in the muffle. Thermocouple wires were welded to the crucible surface. The effects were the same: with the gas flame the surfaces were smooth, with the furnace they were ribbed. A thermocouple was welded to the inside of a crucible. The temperature of the inside of an uncovered crucible in a Meker flame is much lower than the outside, presumably because of radiation to space from the inside of the crucible.
Various heatings using both the welded-on thermocouples and the thermocouple of the muffle, above and below the $1200{ }^{\circ} \mathrm{C}$ of the original observations were made. The differences between figures 4 and 7 and figure 6 cannot be explained by any total over-the-surface difference in temperature. The surface that results is not sensitive to temperature differences as large as the greatest possible error of the measurement.

A tentative explanation has been developed to explain these phenomena. In the muffle furnace the crucible was in a surrounding temperature determined by the surrounding environment, that of the air and of the walls of the furnace, all nearly the same. In the case of the flame of the enriched water gas (a mixture of hydrogen, carbon monoxide, and some hydrocarbon) that emerged from the Meker burner, the chemical reaction takes place all around the crucible. The surface of the crucible itself is part of the flame. Hydrogen dissolves in the hot platinum as it is known to do. When at a point in the surface hydrogen and oxygen atoms unite they emit heat; they cause an atomically limited local elevation of temperature. The platinum atoms at the spot are heated. They tend to scurry along the surface, regardless of the presence of the more stable configurations represented by natural crystal planes until they pop into a temporarily cooler spot in the surface. They smooth the surface, nearly but not quite wiping out the evidences of intracrystalline structures that are the causes of the facelets shown in the figure 4 .

Another heating in a muffle after figure 6 yielded the photomicrograph of figure 7 . The new crystal of figure 6 has moved farther in, cutting into the region of crystal 3 and totally superseding the surfaces of crystals 1 and 2 . The boundary between crystals 3 and 4 has not changed much. 


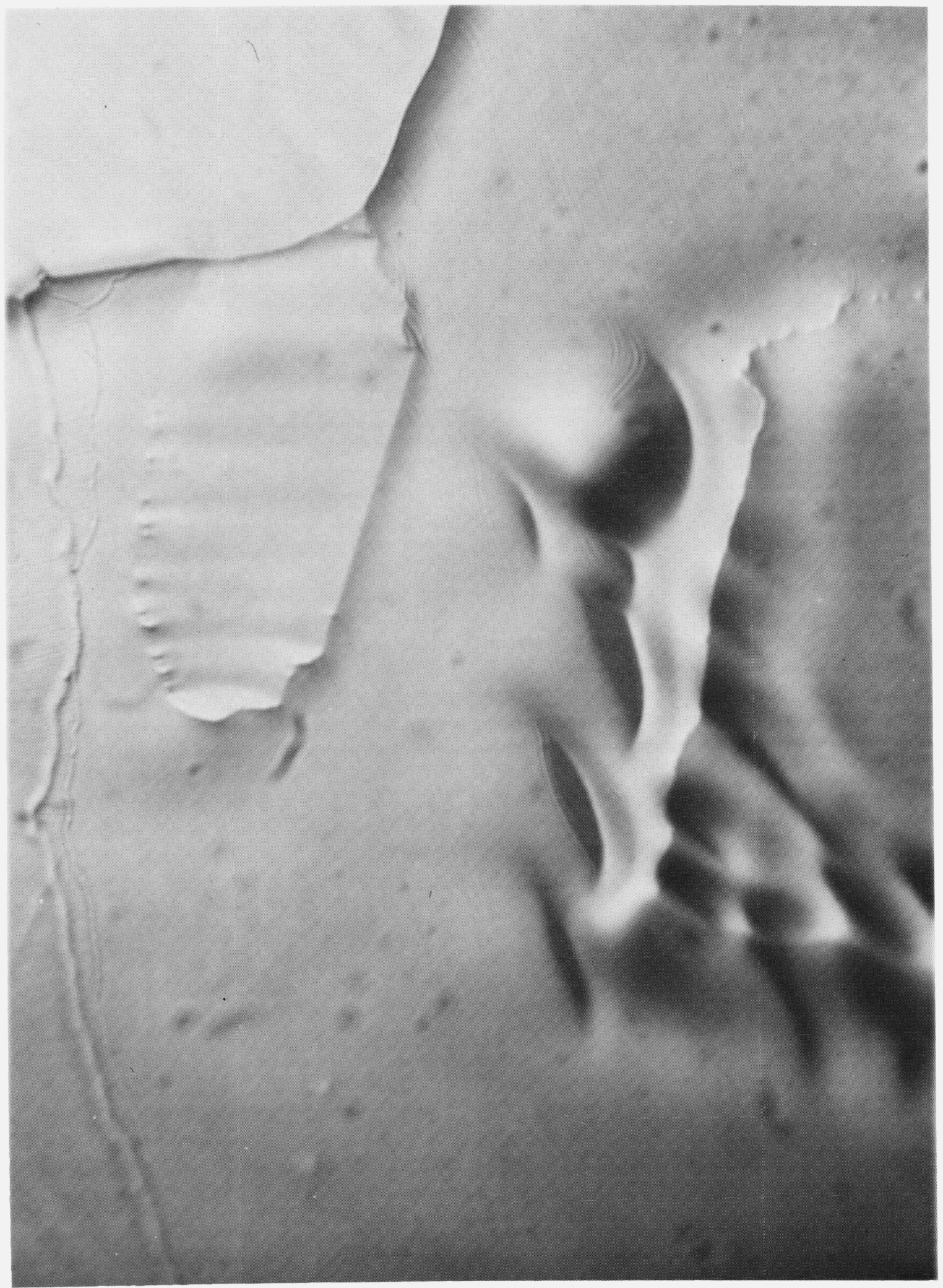

Figure 6. Same area as heated to $1200^{\circ} \mathrm{C}$ in a gas flame. Meker burner fed by carburetted water gas. Same optical conditions. $(\times 2000)$ 


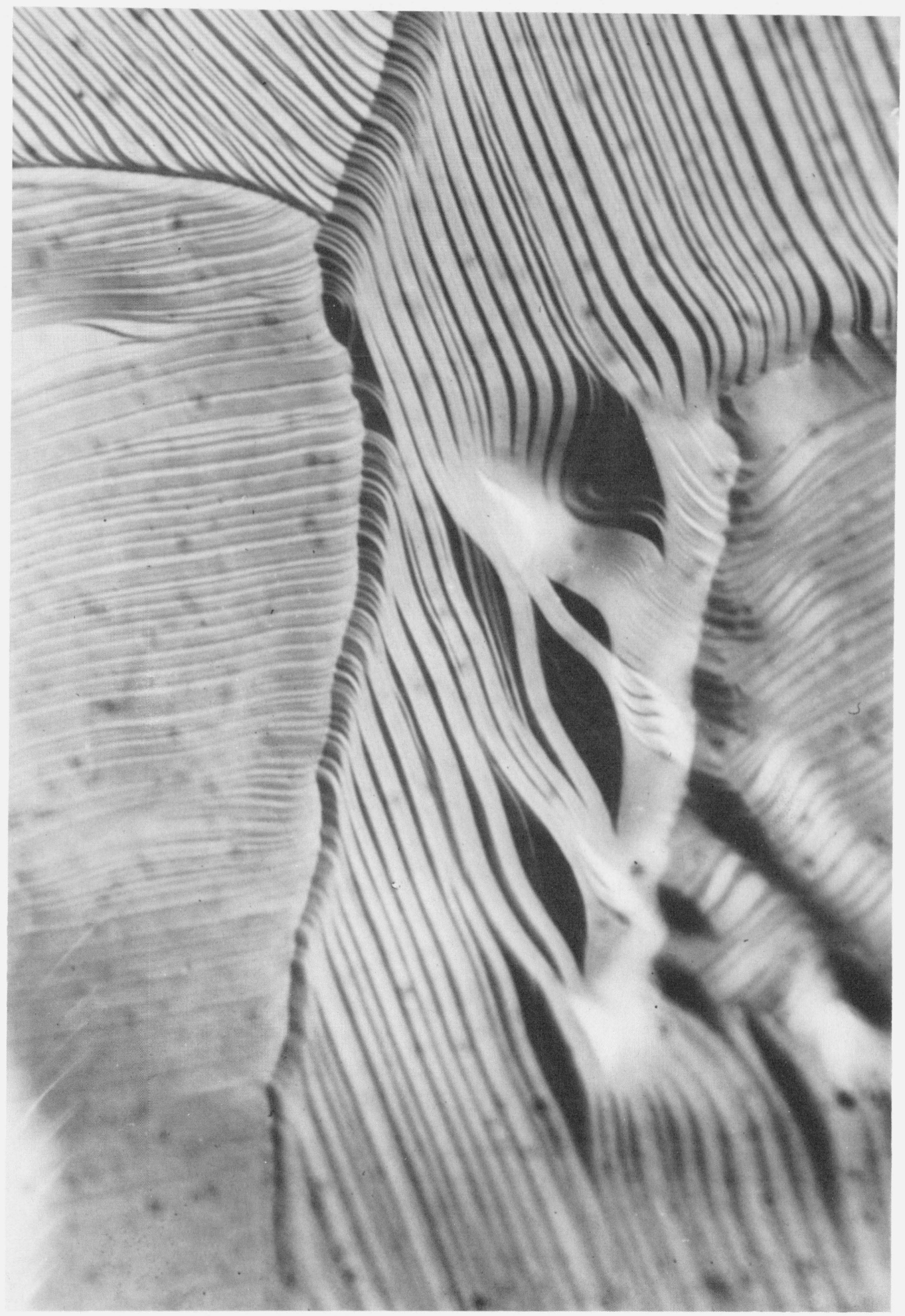

Figure 7. Same area after heating conditions like figure 4. $(\times 2000)$ 
Heating in the muffle has restored the boldly terraced surfaces that were so conspicuous in figure 4 . The decreased spot heating of the platinum surface has restored the more stable configuration.

\section{Recapitulation}

The causes of high iron blanks in standard wet analyses using platinum crucibles were found. The temperature ignition was an essential factor. At such temperatures iron atoms can quickly migrate within the platinum. The studies led to an understanding of the embrittlement and ultimate fracture of those crucibles containing iron which were then subjected to repeated prolonged heatings either in an electric muffle furnace or in a hot gas flame. The crumbling degeneration of crucibles calcined in contact with magnesium pyrophosphate or related substances, while not a topic of the present work, is almost certainly the result of high-temperature hydrogen reduction of the ignited residue to yield a platinum magnesium alloy, a platinum-phosphorus compound, or possibly both.

The magnification $(2000 \times)$ for the photomicrographs shown in figures 4,6 , and 7 would normally be considered excessive (empty magnification). The numerical aperture of both objective and illuminator was 1.32. Ordinarily this would be construed as justifying a maximum enlargement of 1320 - one thousand times the joint numerical aperture. But the diaphragms inserted into both illuminating and viewing systems decrease the resolution in the horizontal direction so that, by the rules, a magnification of only $660 \times$ would seem appropriate.

In the case of figure 4 , a magnification of $660 \times$ leaves the saw-tooth apices of the central crystal so that they can barely be noticed. Even after they are pointed out, one must look closely to see them. Apparently there is more resolution in these photomicrographs than would be anticipated from the half apertures that were employed. The fact that most of the aperture of the illuminating system lies outside the aperature of the viewing system probably means that images are created almost as though both full apertures were used.

The differences in texture of platinum surfaces depending on the method of heating were not understood when this experimental work was done. Consequently any publication describing the work was delayed for many years. Since we are now able to offer a reasonable explanation, we feel justified in presenting the results at this time.

\section{References}

[1] Clarke, F. W. William Francis Hillebrand, National Academy of Science Biographical Memoirs, Vol. 12, (National Academy of Science, Washington, D.C., 1929).

[2] Hillebrand, W. F. Numerous short articles in U.S. Geological Survey Bulletin and the Journal of the American Chemical Society. More notably the books: Analysis of Silicate and Carbonate Rocks, (U.S. Geological Survey Bulletin. No. 305, Washington, D.C.,) 1907, and Analyse der silicat und carbonat-gesteine (Engelmann, Leipzig,) 1910.

[3] Burgess, G. K. and Sale, P. D. Bull. U. S. Bur. Stands., 12, 289 (1915-16), S254.

[4] Wright, F. E. Methods of Petrographic Microscopic Research (Carnegie Institution of Washington, Washington, D.C. 1911). Plato 6, Fig. 3.

[5] Carl Zeiss, Jena. No date.

[6] Merwin, H. E. and Larson, E. S. Am. Jour. Sci. 4th Series 34, 42 (1912).

[7] Barth, Thom. Amer. Mineral. 14, 358 (1929).

[8] Saylor, C. P. Accuracy of microscopical methods for determining refractive index by immersion, J. Res. Nat. Bur. Stand. (U.S.), 15, 277-294 (Sept. 1935).

[9] Williams, R. C. and Wyckoff, R. W. G. Science 101, 594 (1945); J. Appl. Physics 17, 23 (1946). 